\title{
On the multispacecraft determination of periodic surface wave phase speeds and wavelengths
}

\author{
C. Foullon, ${ }^{1}$ C. J. Farrugia, ${ }^{2}$ A. N. Fazakerley, ${ }^{3}$ C. J. Owen, ${ }^{3}$ F. T. Gratton, ${ }^{4}$ \\ and R. B. Torbert ${ }^{2}$ \\ Received 13 December 2009; revised 9 February 2010; accepted 8 March 2010; published 2 September 2010.
}

[1] Observations of surface waves on the magnetopause indicate a wide range of phase velocities and wavelengths. Their multispacecraft analysis allows a more precise determination of wave characteristics than ever before and reveal shortcomings of approximations to the phase speed that take a predetermined fraction of the magnetosheath speed or the average flow velocity in the boundary layer. We show that time lags between two or more spacecraft can give a qualitative upper estimate, and we confirm the unreliability of flow approximations often used by analyzing a few cases. Using two-point distant magnetic field observations and spectral analysis of the tailward magnetic field component, we propose an alternative method to estimate the wavelength and phase speed at a single spacecraft from a statistical fit to the data at the other site.

Citation: Foullon, C., C. J. Farrugia, A. N. Fazakerley, C. J. Owen, F. T. Gratton, and R. B. Torbert (2010), On the multispacecraft determination of periodic surface wave phase speeds and wavelengths, J. Geophys. Res., 115, A09203, doi:10.1029/2009JA015189.

\section{Introduction}

[2] The frequent motions of the magnetopause and of its adjacent boundary layers can be of different origins. Clearly, they may be caused by a variable shocked solar wind plasma impinging on this region. However, they may also be due to inherent instabilities at the boundary itself, brought about for example by flow shears, in the case of the Kelvin-Helmholtz (KH) instability [e.g., Southwood, 1968; Hasegawa, 1975; Farrugia et al., 1998]. The latter mechanism, in particular, may lead in its nonlinear stage to plasma entry to the magnetosphere, by entraining oppositely directed magnetic field lines in the vortical structures, which it gives rise to [e.g., Hasegawa et al., 2004]. Although it has been investigated on multiple levels of time-dependent simulations [e.g., Miura, 1984; Wu, 1986; Manuel and Samson, 1993; Thomas and Winske, 1993; Fujimoto and Terasawa, 1994], this mechanism needs a violation of the frozen-in law or magnetic reconnection triggered in $\mathrm{KH}$ vortices [e.g., Otto and Fairfield, 2000; Nykyri and Otto, 2004]. Wave characteristics, such as the sizes of $\mathrm{KH}$ vortices, and other derived quantities may be of physical importance, notably via comparison with theoretical and numerical models, to

\footnotetext{
${ }^{1}$ Center for Fusion, Space and Astrophysics, Department of Physics, University of Warwick, Coventry, UK.

${ }^{2}$ Space Science Center, Department of Physics, University of New Hampshire, Durham, New Hampshire, USA.

${ }^{3}$ Mullard Space Science Laboratory, University College London, Dorking, UK.

${ }^{4}$ Instituto de Física del Plasma, CONICET, Universidad de Buenos Aires, Buenos Aires, Argentina.

Copyright 2010 by the American Geophysical Union. 0148-0227/10/2009JA015189
}

elucidate the conditions leading to the waves' formation, their possible non-linear development and their role in the plasma entry to the magnetosphere. For these reasons, reliable estimates of the wave characteristics are important.

[3] Quoted values of surface wave observations on the magnetopause indicate that their periods are typically in the Pc5 $(1-10 \mathrm{mHz})$ range, with a wide range of phase velocities (varying from about $60 \mathrm{~km} \mathrm{~s}^{-1}$ [see Foullon et al., 2008] to $350 \mathrm{~km} \mathrm{~s}^{-1}$ [see Chen et al., 1993]) and a similar spread in wavelengths (from $2 \mathrm{R}_{\mathrm{E}}$ [see Foullon et al., 2008] to a few tens of $\mathrm{R}_{\mathrm{E}}$ [see Hones et al., 1981]). Arguably, a key factor controlling the wavelengths, $\lambda$, is the distance of the observing site from the subsolar point because the magnetosheath flow speed picks up as one moves tailward, leading to $\lambda$-stretching effect, and the magnetic field strength decreases along the magnetopause, implying that all gyroradii increase. In addition, KH surface waves in the magnetospheric context can become nonlinear while propagating down the tail. Nonlinear effects have been invoked to account for (1) wavelengths of a few $R_{E}$ typically observed on the magnetopause, which, as argued by Belmont and Chanteur [1989], are much longer than those predicted by linear theory; (2) the tailward steepening of the KH leading fronts observed with Cluster [Owen et al., 2004; Foullon et al., 2008], consistent with the growing phase of $\mathrm{KH}$ waves [De Keyser et al., 2005] (note that the inverse dependence found between the boundary layer thickness and the tailward steepening of the leading edge [Foullon et al., 2008] suggest that this effect is affected by changing conditions of the medium in which the waves propagate); (3) the presence of vortices, a phenomenon supported by the interpretation of data in single or multispacecraft analyses [Hones et al., 1981; Saunders et al., 1983; Fairfield et al., 2000; Hasegawa et al., 
2004; Fairfield et al., 2007]; (4) an inverse dependence between the interplanetary magnetic field (IMF) clock angle and the wavelength at the flank [Foullon et al., 2008] or the period of the magnetospheric micropulsations these waves excite [Farrugia et al., 2000], which confirms the significance of source regions and nonlinear development for interpreting observations of remotely generated $\mathrm{KH}$ waves.

[4] In the simplest linear magnetohydrodynamic (MHD) description, the phase speed of a $\mathrm{KH}$ wave propagating with wave vector $\mathbf{k}$ in an ideal incompressible plasma, with a discontinuous velocity shear layer and assuming the layer to be infinitely thin [Chandrasekhar, 1961; Hasegawa, 1975], is

$$
v_{p}=\frac{n_{1} \mathbf{k} \cdot \mathbf{V}_{\mathbf{1}}+n_{2} \mathbf{k} \cdot \mathbf{V}_{\mathbf{2}}}{k\left(n_{1}+n_{2}\right)} .
$$

Here the indices refer to the two plasma environments on either side of the boundary, $n$ is the plasma number density, $\mathbf{V}$ is the plasma flow velocity; $\mathbf{k}$ and $\mathbf{V}$ (and the magnetic field vector, $\mathbf{B}$, not present in equation (1)) are all tangential to the layer. Equation (1) is the real part, $v_{p}=$ $\omega / k$, in the dispersion relation associated with complex roots, $\sigma=\omega+i \gamma$, for perturbations along the boundary surface, i.e., expi(k.r $-\sigma t)$. The imaginary part (not shown), which corresponds to the growth rate of the instability, $\gamma$, is a competition between the destabilizing effects of velocity gradient or shear, $\left|\mathbf{V}_{\mathbf{1}}-\mathbf{V}_{\mathbf{2}}\right|$, and the stabilizing effects from magnetic tension forces; the $\mathrm{KH}$ instability criterion is met for $\gamma>0$. Several observations show a set of $\mathbf{k}$ directions of predominantly plane disturbances consistent with the conditions predicted by this ideal $\mathrm{KH}$ instability criterion [e.g., Ogilvie and Fitzenreiter, 1989; Owen et al., 2004].

[5] While this treatment is only approximate and qualitative as applied to the magnetospheric boundary and to nonlinear (vortical) and multiwavelength structures, the dimensions of those structures may be better estimated with the group velocity, which is the energy propagation velocity of waves of different wavelengths and propagation directions. Considering the convectively unstable surface mode [e.g., Mills et al., 2000; Wright et al., 2000], perturbations along the boundary surface, while growing, are convected downstream with the phase velocity $v_{p}(\mathbf{k}) \mathbf{k}$ and the group velocity, $\mathbf{v}_{\mathbf{g}}$, which from equation (1) corresponds to

$$
\mathbf{v}_{\mathbf{g}}=\frac{\partial \omega}{\partial \mathbf{k}}=\frac{n_{1} \mathbf{V}_{\mathbf{1}}+n_{2} \mathbf{V}_{\mathbf{2}}}{n_{1}+n_{2}} .
$$

The group velocity is the propagation velocity of the wave envelope. Thus if there is a case for a coherent, nondispersive structure or wave envelope propagating in a particular direction, then the speed, $V_{k}$, that connects its leading and trailing front (i.e., speed of the wave envelope) is as meaningful as the group speed for calculating the wavelength of that structure. In this work, such observational nondispersive 'phase speeds' and associated propagation directions are discussed.

[6] Besides the stream of flow velocity, other factors may control $\lambda$, including (1) the thickness of the boundary layer [e.g., Walker, 1981; Miura and Pritchett, 1982], (2) the nonlinear development of dominant structures [e.g., Belmont and Chanteur, 1989; Miura, 1999a, 1999b], and (3) the global magnetospheric geometry of unstable source regions controlled by the IMF orientation [e.g., Farrugia et al., 1998; Foullon et al., 2008]. All those factors have therefore a direct effect on $V_{k}$.

[7] Despite those latest theoretical developments, most observational, as well as theoretical works, assume some 'flow approximations', which may be seen to correspond to limiting cases to equation (2). Focusing on the $\mathrm{KH}$ waves, we first outline the advantages and results of multispacecraft timing, using between 2 and 4 spacecraft. We then compare and discuss some flow approximations often used, where we note clear disagreements, and thus propose alternative consistency checks to improve the estimates.

\section{Surface Wave Phase Speeds From Multispacecraft Observations}

[8] Triangulation or four-spacecraft timing analysis [Russell et al., 1983; Schwartz, 1998] has been applied to our knowledge in two Cluster studies of surface waves, that by Owen et al. [2004] and that by Foullon et al. [2008], on the dawn and dusk flanks respectively. The waves studied have a sawtooth shape, which they retain as they propagate past Cluster (within a relatively short time interval), at an epoch when the four satellites have separations of $\sim 2000 \mathrm{~km}$. Such conditions are very appropriate for estimating their phase speeds, and consequently their wavelengths, via the method proposed by Foullon et al. [2008] (see illustration by Foullon et al. [2009] and also a more approximative variant in the work of Owen et al. [2004]). This method uses aggregate results from four-spacecraft timing analysis applied to a pair of bounding surfaces.

[9] Figure 1 explains a 2-D version of the geometries considered. The wavefront is shown by the vertical dotted line and so the propagation direction $\mathbf{k}$ is along the horizontal direction. The separation distance along $\mathbf{k}$ between two samplings of the wavefront in a $1 \mathrm{~s}$ time interval corresponds therefore to the value of the phase speed, $V_{k}$ (in $\mathrm{km} \mathrm{s}^{-1}$ ). For a surface (plain line) inclined with respect to the wavefront, the separation distance is the same (Figure 1a). If the two sampling spacecraft are not aligned along $\mathbf{k}$, then the distance between the two samplings of what appears to be the same inclined surface (with the same given phase) is covered in $1 \mathrm{~s}$ with a larger apparent speed (Figure 1b). This later configuration corresponds to speeds along normals of bounding surfaces, typically inclined with respect to the wavefront. In a 3-D geometry, such speeds and surface normals can be obtained via four-spacecraft timing and the speeds are still larger than the periodic surface wave phase speed. The two-point and four-point measurements have the same problem of the apparent speed. The periodic surface wave phase speed is obtained via 'deprojection' along the wave propagation direction.

[10] The former configuration (Figure 1a) corresponds to the case of two spacecraft aligned along the magnetospheric boundary and presumably along $\mathbf{k}$, with the phase speed obtained by taking the spacecraft separation distance divided by the time lag between the two observations, e.g., ISEE-1 and ISEE-2 [Hones et al., 1981; Chen et al., 1993]. Even with the scale size of four spacecraft (tetrahedral) configuration (more precisely the tetrahedron scale size along the average direction tangential to the boundary), it is 


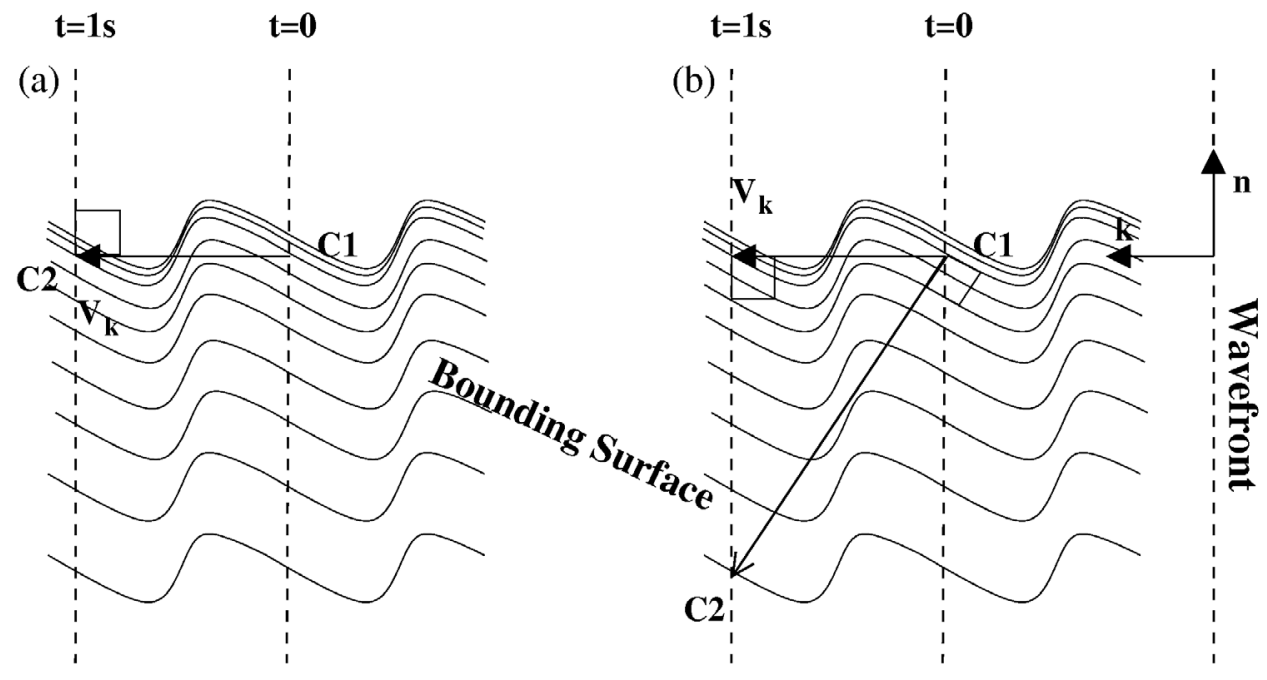

Figure 1. Surface wave propagating in the $\mathbf{k}$ direction with phase speed, $V_{k}$, showing bounding surfaces sampled by spacecraft $\mathrm{C} 1$ at time $\mathrm{t}=0$ and $\mathrm{C} 2$ at $\mathrm{t}=1 \mathrm{~s}$, respectively, (a) without and (b) with inclination of the two-spacecraft direction with respect to $\mathbf{k}$.

possible to give an optimal estimate of the phase speed by considering the largest time lag between spacecraft. For instance, taking the ratio of the Cluster scale size of 2000 $\mathrm{km}$ over the largest time lag of $30 \mathrm{~s}$ [see Foullon et al., 2008], yields a value of about $67 \mathrm{~km} \mathrm{~s}^{-1}$, in good agreement with the more accurate results derived from fourspacecraft timing analysis. This can therefore be used to give a qualitative and immediate validation of the more comprehensive method. As illustrated in Figure 1b, the 'in-between spacecraft' speed may become an upper estimate to the real phase speed.

[11] Both Cluster studies of surface waves [Owen et al., 2004; Foullon et al., 2008] refer to similar distances from the subsolar point (with spacecraft located at $X_{G S M} \approx$ $[-3,-6] \mathrm{R}_{\mathrm{E}}$, on opposite sides of noon and at low geomagnetic latitudes) and indicate phase speeds $\left(50-90 \mathrm{~km} \mathrm{~s}^{-1}\right)$ and wavelengths $\left(2-3.4 \mathrm{R}_{\mathrm{E}}\right)$ in the lower range compared to other reports in the same locales. Accurate determination of the required time lags for a main front passage can be achieved via the use of both (1) plasma and (2) magnetic field data sets. First, plasma data sets that display sharp transitions between adjacent plasma environments have to be available on four spacecraft, e.g., the electron temperature from PEACE [Owen et al., 2004; Foullon et al., 2008]. Second, to deal with magnetic field data sets, which are more highly perturbed, Foullon et al. [2008] proposed a multiscale approach to select recurring local extrema, for different scales or levels of smoothing, in the time derivative of a magnetic field (sunward) component. The reliability of those timings is further cross-checked with the use of error estimates on the combined (plasma and magnetic field) results. For the dusk event, the errors on the phase speed range from 3 to $28 \mathrm{~km} \mathrm{~s}^{-1}$. The phase speed from the Cluster results represents therefore a small fraction (a third or less) of the magnetosheath flow speed, $V_{s}\left(V_{s} \sim 260 \mathrm{~km} \mathrm{~s}^{-1}\right.$ in the work of Foullon et al. [2008]).

[12] In the case of surface waves, the timings obtained locate the same phase in the waveform but not necessarily for the same isosurface (or discontinuity). The application of four-spacecraft timing analysis in this case thus differs from the case of shocks [e.g., Foullon et al., 2007], where the propagation along the normal can be expected. Nevertheless, the technique can be applied as long as the plane surface hypothesis is valid, i.e., a quasi-planar subsection of the waveform is sampled, when the spacecraft separation is relatively small. This can be the case also in the presence of rolled-up vortices, for detected planar disturbances that embed the vortices. Finally, the effect of overall motions in response to varying conditions, which may interfere with the measurements, can be mitigated by taking the average of phase speeds and propagation directions between pairs of inward and outward motions [Foullon et al., 2008]. This average can also result in an approximate direction of propagation, if the $\mathbf{k}$ direction changes notably across the boundary layer. The directions of propagation obtained in both Cluster studies are found to be perpendicular to an average external magnetic field direction, rather than being related to a magnetosheath flow component, suggesting that the wave propagation direction may adjust to the bending of field lines developed by the KH waves [Foullon et al., 2010]. Comparisons with the single-spacecraft method introduced by De Keyser and Roth [2003] would be desirable. This latter method estimates the surface wave phase speed for which the flow in a frame comoving with the wave is parallel to the density gradient (usually in the boundary layer).

\section{Flow Approximations Versus Estimates From Time Lags}

[13] Another multispacecraft approach was proposed by De Keyser et al. [2004] [see also De Keyser et al., 2005]. It uses an empirical reconstruction technique to obtain a $2 \mathrm{D}$ picture of the surface waves in a reference frame that slides along the boundary with the wave. Here the phase speed of the wave is assumed. For an event they examined on 25 November 2001, for instance, the phase speed is taken to 
be equal to one half of the magnetosheath speed, i.e., $V_{k}=$ $200 \mathrm{~km} \mathrm{~s}^{-1}$. However, the latter value does not concur with an (upper) estimate of about $100 \mathrm{~km} \mathrm{~s}^{-1}$, which we infer from the $2500 \mathrm{~km}$ scale size of the spacecraft separation and the $25 \mathrm{~s}$ maximum time lags for the same event (seen in EFW densities [De Keyser et al., 2005, Figure 8.37]).

[14] Many researchers stipulate the phase speed as a given fraction of $V_{s}$. This can lead to erroneous results and we are here trying to show how to improve these estimates. There are 'anomalous' cases of magnetopause waves with opposite (sunward) steepened edges reported from two-spacecraft observations by ISEE-1 and ISEE-2 [Chen et al., 1993; Chen and Kivelson, 1993]. These anomalous waves appear to form in association with strong magnetosheath plasma acceleration [Lavraud et al., 2007]. Note that such conditions may lead to both $\mathrm{KH}$ and anomalous surface waves. The example of $1 / 2$ magnetosheath speed found in one case of surface waves [Chen et al., 1993] was later applied to other events without distinction. This was done first in another case of anomalous surface wave [Chen and Kivelson, 1993], yielding an estimate of $100 \mathrm{~km} \mathrm{~s}^{-1}$. This value seems also overestimated given that the observations indicate a time lag of $10 \mathrm{~s}$ between ISEE-1 and ISEE-2 (as seen in the work of Chen and Kivelson [1993, Figure 3] that are separated by about $420 \mathrm{~km}$, i.e., a phase speed no greater than about $42 \mathrm{~km} \mathrm{~s}^{-1}$.

[15] Another ad hoc approximation is to take the average mean bulk flow velocity in the boundary layer as estimate for the phase speed. Several properties of the magnetic and velocity fields show gradually varying profiles across the layer [e.g., Phan et al., 1997; Foullon et al., 2008]. Like the group speed in equation (2), the flow velocity in the boundary layer is taken on average to be intermediate between the magnetosheath and the (stagnant) magnetospheric velocities, despite evidence of plasma acceleration presumably signaling the presence of rolled-up $\mathrm{KH}$ waves. For a subset of the 20 November 2001 event [Foullon et al., 2008 , interval D], the average flow velocity along the $X_{G S M}$ direction of $212 \mathrm{~km} \mathrm{~s}^{-1}$ [Hasegawa, 2009] is twice as large as the phase speed deprojected in the $X_{G S M}$ direction, i.e., $100 \pm 11 \mathrm{~km} \mathrm{~s}^{-1}\left(V_{k}=85 \pm 9 \mathrm{~km} \mathrm{~s}^{-1}\right.$ from Foullon et al. [2008]). For coalignment of the time series in the comoving frame of the $\mathrm{KH}$ wave in the $X_{G S M}$ direction [Hasegawa et al., 2004], this apparent phase speed discrepancy on the Cluster $2000 \mathrm{~km}$ scale size can only lead to a spatial or temporal shift correction between time series of about $(2000 \times(1 / 100-1 / 212) \sim) 11 \mathrm{~s}$ at most. Therefore this inaccuracy does not noticeably affect the apparent good alignment of magnetic and velocity fluctuations [Hasegawa et al., 2004, Figure 2] with period of $215 \mathrm{~s}$ [Foullon et al., 2008]. As a result, it is unlikely to change the observations of higher densities intruding in lower density regions, which is central to the interpretation of rolled-up vortices [Hasegawa et al., 2004]. However, the inaccuracy of the phase speed by a factor greater than two has a direct effect on the wavelength determination.

\section{Wavelength Proxy From Two-Point Distant Comparison}

[16] Multipoint in situ observations are exceptionally favorable for data analysis and the characterization of $\mathrm{KH}$ activity. However, it becomes more challenging to crosscorrelate data sets at increasing separation distances due to the misalignment of the spacecraft along $\mathbf{k}$. For illustration we use the 20 November 2001 event, with two low-latitude observations separated by several $\mathrm{R}_{\mathrm{E}}$ along the dusk flank magnetopause, as shown in Figure $4 \mathrm{a}$, with Geotail on the dayside and Cluster tailward of the terminator. The twopoint observations may be compared at the time of relatively large IMF clock angle $\left(41 \pm 18^{\circ}\right.$ between 1804 and 2004 UT [Foullon et al., 2008, interval C]).

[17] Figures 2 and 3 illustrate the spectral analysis of the wave activity at Geotail and Cluster, using magnetic field data from the Magnetic Field investigation (MGF) aboard Geotail [Kokubun et al., 1994] and the Fluxgate Magnetometer (FGM) aboard Cluster C2 [Balogh et al., 2001], with time cadence of about $3 \mathrm{~s}$ and $0.2 \mathrm{~s}$, respectively. For comparison between the two data sets, the magnetic field vectors $\mathbf{B}$ are first projected into a boundary coordinate system $(\mathbf{l}, \mathbf{m}, \mathbf{n}): \mathbf{n}$ is chosen to be the normal to a model magnetopause [Roelof and Sibeck, 1993] scaled to the respective spacecraft position (at 1918 UT, for Geotail, $\mathbf{n}=[0.833,0.548,0.075]$ and at $1904 \mathrm{UT}$, for Cluster, $\mathbf{n}=$ $[0.504,0.855,-0.120]$, given in the GSM system), $\mathbf{m}=\mathbf{n} \times$ $\mathbf{z}_{\mathrm{GSM}} / \mathbf{| n} \times \mathbf{z}_{\mathrm{GSM}} \mid$ and $\mathbf{l}=\mathbf{m} \times \mathbf{n}$ [Elphic and Russell, 1979]. As shown in Figures $2 \mathrm{a}-2 \mathrm{c}$ and Figures $3 \mathrm{a}-3 \mathrm{c}$, the components are then normalized and interpolated to a regular $1-\mathrm{s}$ cadence time array.

[18] For the whole event at Cluster, we have shown that the magnetic wave activity is characterized with the largest component $B(m)$ of the magnetic field and that amplitude variations in this tailward component ( $m$ pointing sunward on the dusk flank) relate to $\lambda$ changes (due to the tailward component stretching in the wave propagation direction). $B(m)$ is also the component with the largest variations at Geotail, which was in the boundary layer between 1918 and 1955 UT (indicated between vertical dotted lines in Figures $2 \mathrm{~b}$ and $3 \mathrm{~b}$ ). According to our cross-correlation analysis of the magnetosheath clock angle adjacent to the magnetopause, there is less than 5 min propagation delay along streamlines (adjacent to the magnetopause) from the dayside magnetosheath near Geotail to the nightside magnetosheath near Cluster [Foullon et al., 2008]. Since the wave propagation delay in the boundary layer is expected to be even shorter, the time frame for comparison between the two sets of perturbations is valid.

[19] Prior to spectral analysis, we detrend the time series $B(m) /|B|$ in a wavelet 'à trous' decomposition, in order to filter out the low frequencies presumably associated with solar wind-driven variations. The trend is overlaid for each time series in Figures $2 b$ and $3 b$. Figures $2 d$ and $3 d$ show the Lomb-Scargle periodogram, normalized Fourier power spectrum, and normalized global (time-averaged) Morlet wavelet $(\mathrm{GW})$ spectrum for the detrended time series $\delta(B(m) /|B|)$. In the range $100-250 \mathrm{~s}(4-10 \mathrm{mHz})$, we can distinguish power spectral peaks at periodicities near $144.2 \mathrm{~s}$ at Geotail and $187 \mathrm{~s}$ at Cluster, which are consistent with the sizes of individual oscillatory structures, whose amplitudes dominate in the time series when inspected visually. As noted by Foullon et al. [2008], a secondary periodicity $(301 \pm 13 \mathrm{~s})$ is present in the Cluster time series; signals above the periodogram confidence level [Bai and Cliver, 1990] are also present near those periods in the Geotail time series. 

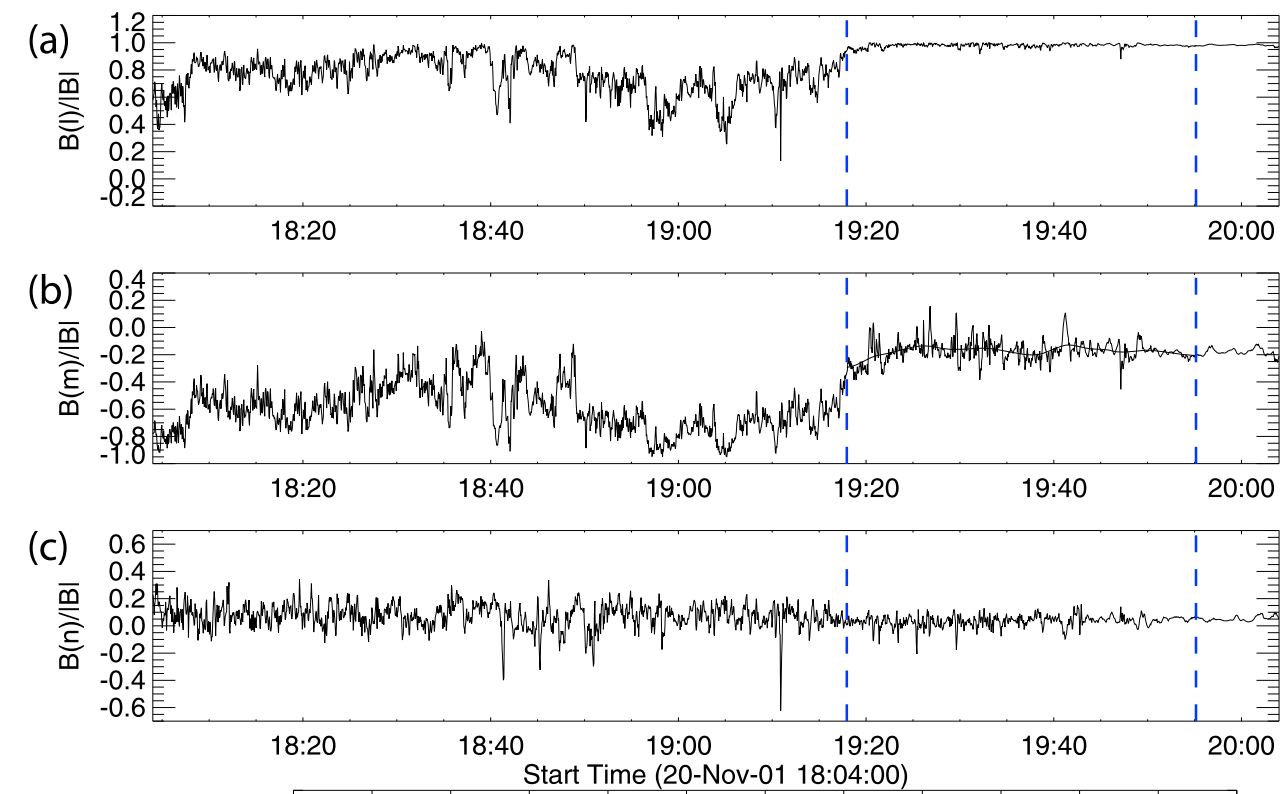

(d)

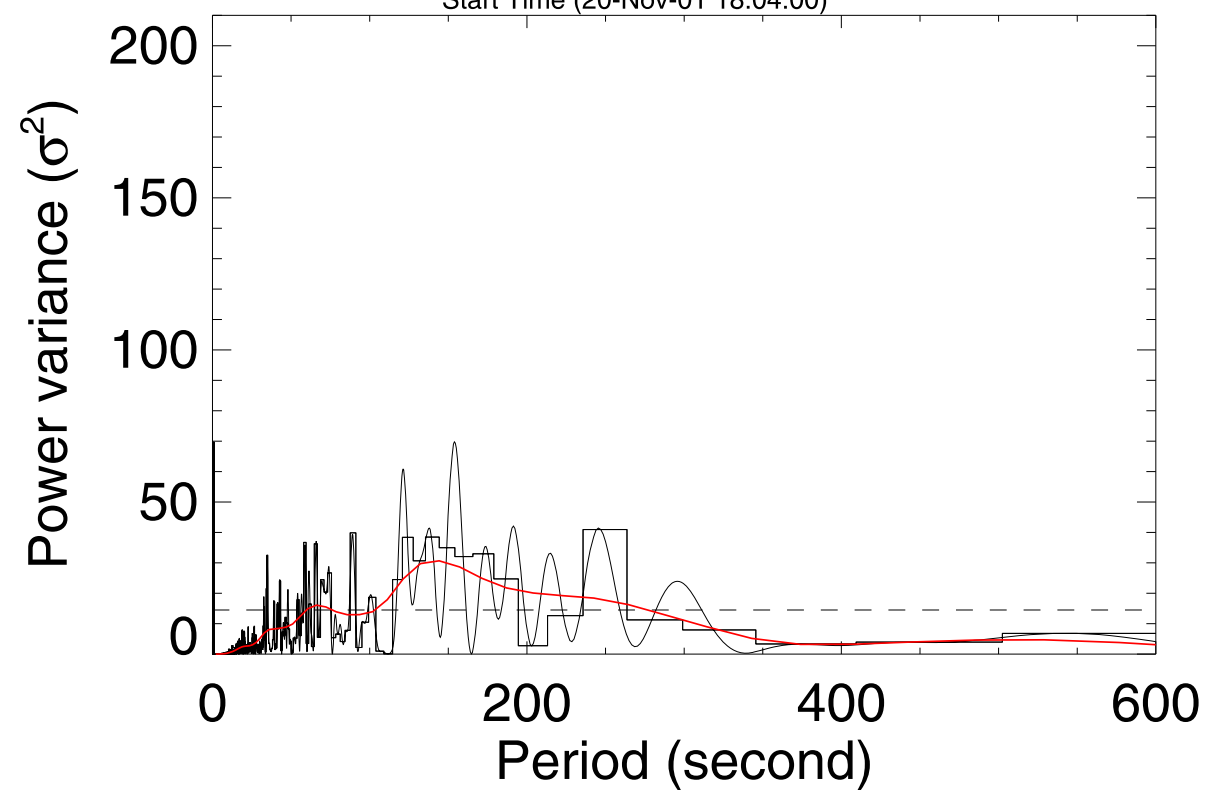

Figure 2. Magnetic wave activity at Geotail in the interval between 1804 and 2004 UT on 20 November 2001. (a-c) Normalized magnetic field time series $B /|B|$, projected into a $l m n$ coordinate system; a trend is overlaid on the $\mathbf{m}$ component, $B(m) /|B|$. Vertical dashed lines mark interval in the boundary layer. (d) Lomb-Scargle periodogram (thin solid), normalized Fourier power spectrum (histogram mode) and normalized global wavelet spectrum (thick red) on the detrended time series $\delta(B(m) /|B|)$. The horizontal dashed line is the $95 \%$ confidence level for the periodogram.

[20] Compared to the dominant signals, the secondary signals at Cluster appears with less power in the Fourier and periodogram spectra but with more power in the GW power spectrum. Despite this difference, to keep in mind, the GW spectrum gives a smoothed version of the Fourier power spectrum [Torrence and Compo, 1998]. Thus to facilitate the comparison between Geotail and Cluster spectra, Figure $4 \mathrm{~b}$ presents the GW spectra together but without normalization. There is a tenfold increase in power at Cluster (interval C) with respect to Geotail (for the interval in the boundary layer). Figure $4 \mathrm{~b}$ shows also the $43 \mathrm{~s}$ increase in periods from $144.2 \mathrm{~s}$ at Geotail to $187 \mathrm{~s}$ at Cluster. While the data may be interpreted as a growth of activity from Geotail to Cluster, i.e., the $\lambda$ of the structures increases, due to an increasing speed of propagation, these distant observations may not be necessarily related to each other. Geotail is near the equator and close to the stagnation region, while the wave activity at Cluster is likely to originate from a position intermediate between Geotail and Cluster (as seen from the subsolar point), since the IMF is substantially away from the Northward direction (see Foullon et al. [2008], KH unstable regions). 

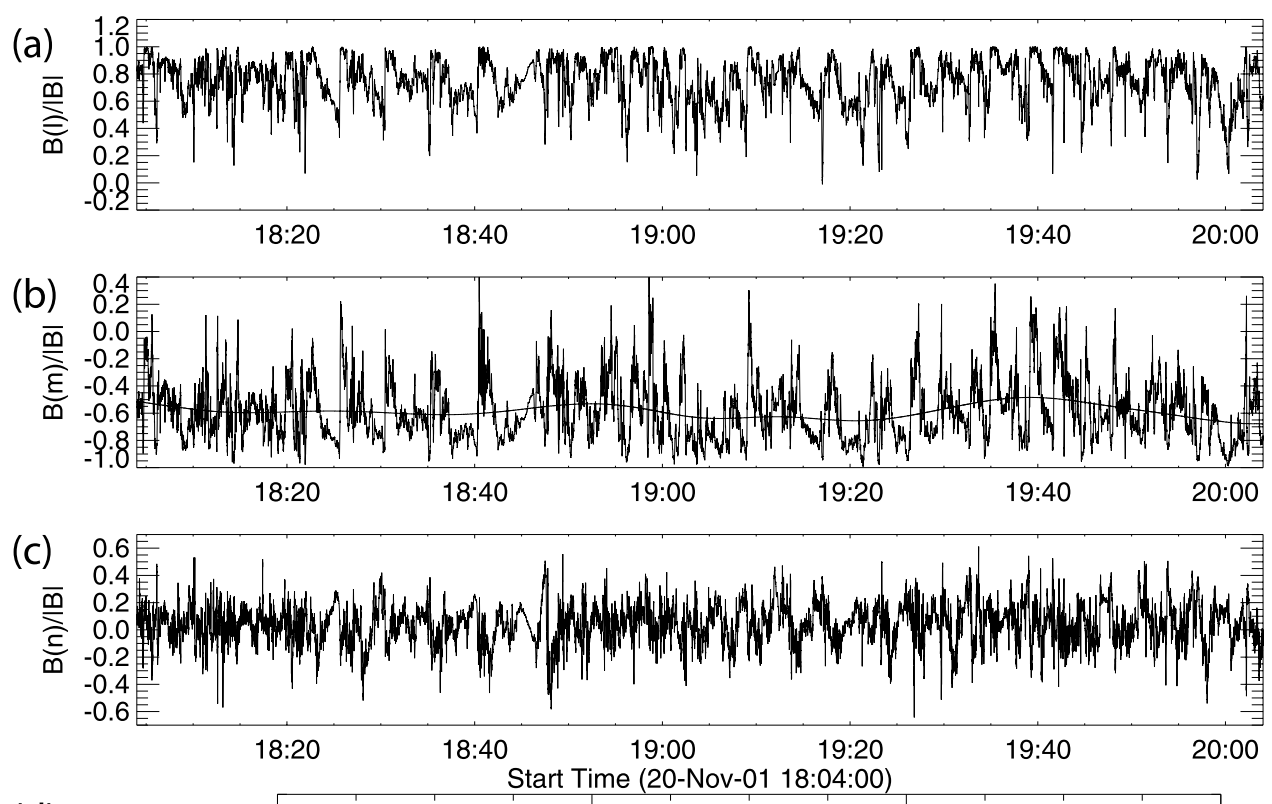

(d)

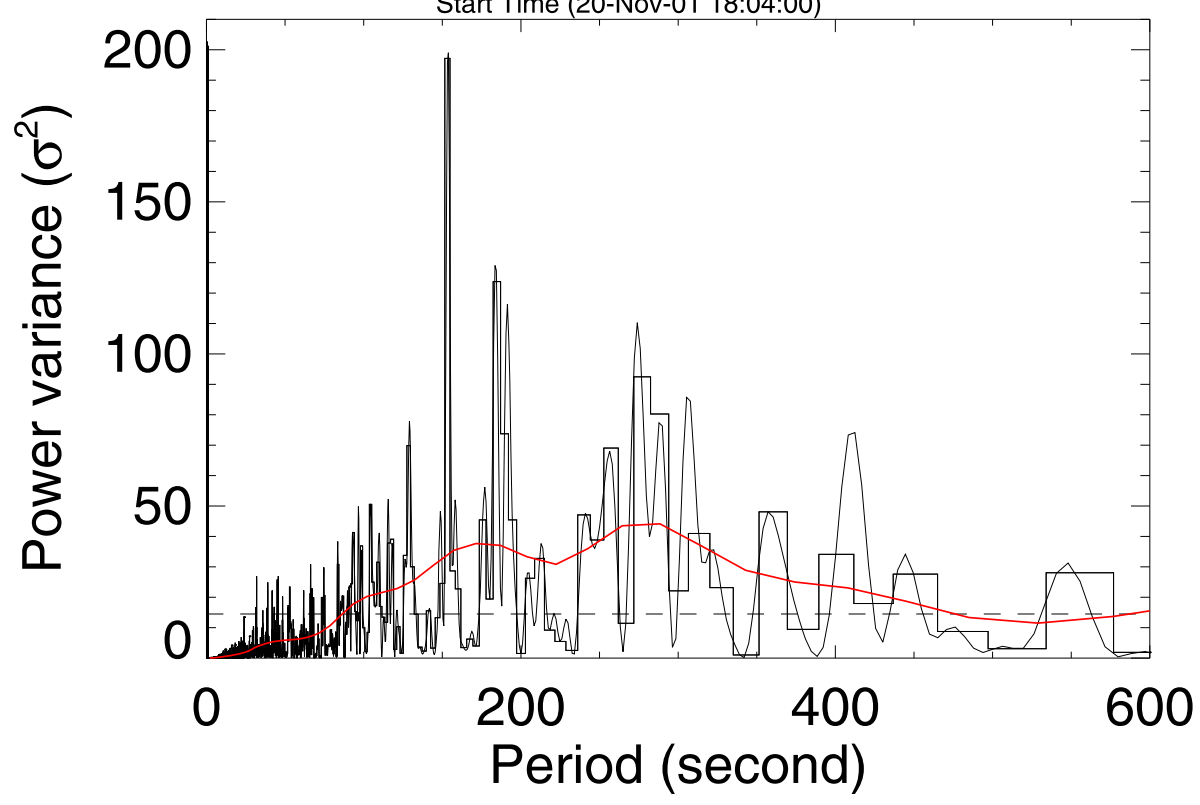

Figure 3. Magnetic wave activity at Cluster $\mathrm{C} 2$ in the same interval and same format as in Figure 2.

[21] Nevertheless, using the spectral power as proxy for $\lambda$, we can infer the relation between the two parameters at Cluster and apply this relation in order to obtain the $\lambda$ at Geotail. A statistical fit at Cluster can be obtained thanks to various levels of IMF clock angles, which control $\lambda$ [Foullon et al., 2008]. Amplitude variations in $B_{m}$ are assumed to depend on $\lambda$, whatever the nature and origin of the perturbations, so that the wave activities from sites, which are not necessarily aligned in a given propagation path, can still be compared. Figure $4 \mathrm{c}$ is a plot of the global wavelet maximum power, $P_{\mathrm{GW}}$, in the range $100-250 \mathrm{~s}$ (4-10 mHz), versus the wavelengths. The Cluster $\lambda$ (upper symbols in black) are obtained by combining results from four-spacecraft timing and spectral analysis. The different symbols correspond to the various levels of IMF clock angles, with positive values around (A: diamond) $36^{\circ}$,
(B: square) $12^{\circ},\left(\mathrm{C}:\right.$ triangle) $41^{\circ},\left(\mathrm{D}:\right.$ circle) $19^{\circ}$ and (E: asterisk) $62^{\circ}$, corresponding to time intervals with relatively small variations of the solar wind speed and density between them. The best fit to the data points at Cluster, using the linear relation

$$
\lambda\left(P_{\mathrm{GW}}\right)=\lambda_{0}+A \log \left(P_{\mathrm{GW}}\right),
$$

with $\lambda$ in km and $P_{\mathrm{GW}}$ in $\mathrm{Hz}^{-1}$, is obtained for $\lambda_{0}=11860 \pm$ $471 \mathrm{~km}$ and $A=1739 \pm 487 \mathrm{~km}$. This relation is used to estimate the $\lambda$ at Geotail (lower triangle in red), i.e., $7253 \pm$ $690 \mathrm{~km}$. From the dominant spectral period, we infer a propagation speed of the perturbations at Geotail of $50 \pm$ $5 \mathrm{~km} \mathrm{~s}^{-1}$. This value is slightly lower but close to the average ion velocity projected in the $\mathbf{m}$ direction of the local 

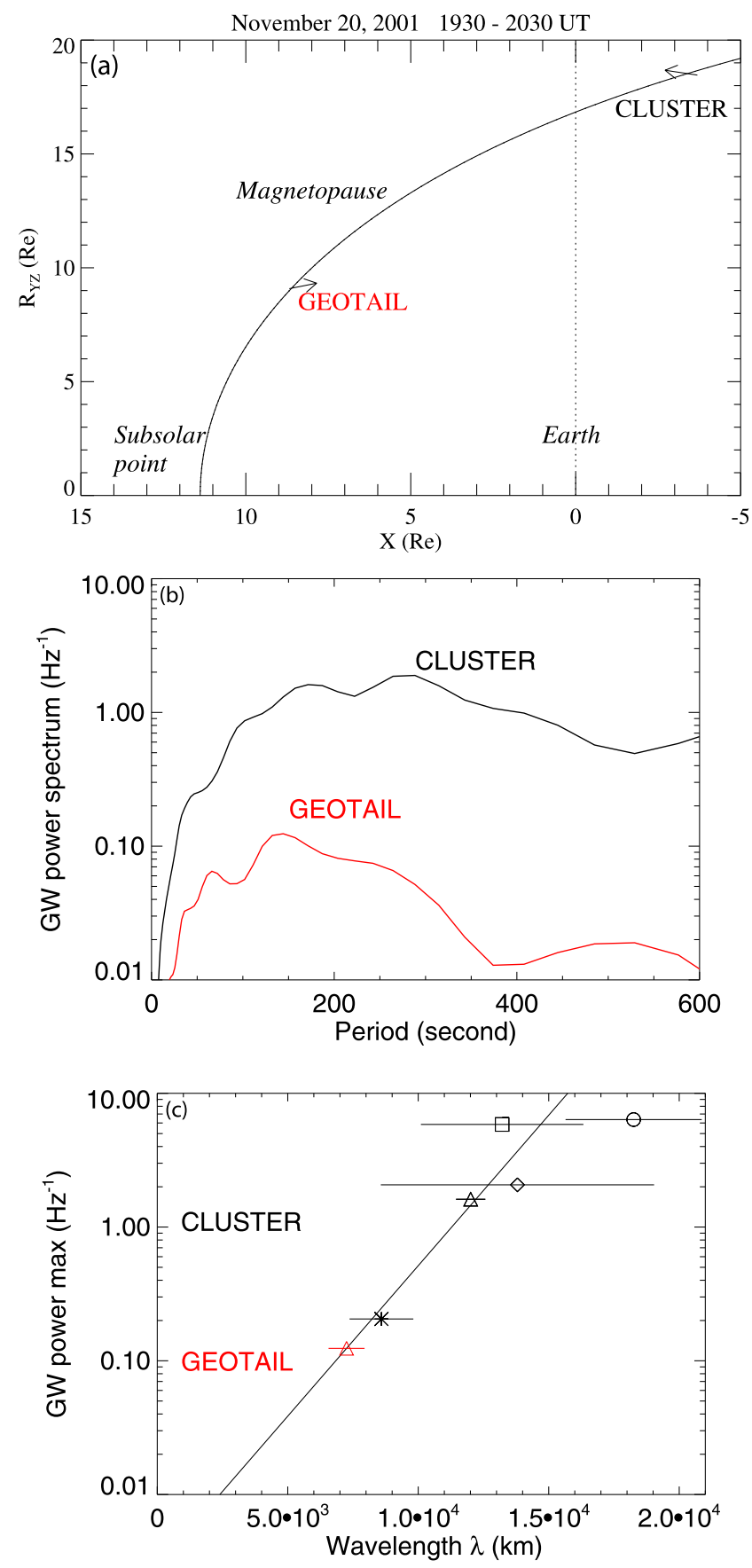

Figure 4. Event of 20 November 2001. (a) Orbit view of Geotail on the dayside and Cluster tailward in a radial cross-section of the dusk magnetosphere. (b) Global wavelet power spectra for the detrended and normalized time series $\delta B(m) /|B|$ at Cluster (black) and Geotail (red). (c) Global wavelet maximum power in the range 100-250 s (4-10 mHz), versus the wavelengths. See text for details.

boundary coordinate system, namely, $57 \mathrm{~km} \mathrm{~s}^{-1}$ in absolute value. Adding error estimates on the spectral power at Geotail (e.g., due to the low-frequency filtering) may slightly broaden the range of $\lambda$ and the propagation speed of the perturbations. This indicates that the perturbations at Geotail are likely to be advected with the local flow. We note that the value obtained is much lower than the $115 \mathrm{~km} \mathrm{~s}^{-1}$ estimate given by Hasegawa et al. [2009] (based on flow approximations).

\section{Conclusions}

[22] Although they represent a modest sample of the observations analyzed over the past, the phase speeds derived so far with Cluster yield values of a $1 / 3$ or less of the magnetosheath flow speed or the average flow speed in the boundary layer. Time lags between two or more spacecraft can give a qualitative upper estimate of the phase speed. Cross-examination of a few cases confirm that the flow approximations often used are generally too large. Such inaccuracies may lead to mistakes in the characterization of the different types of waves, in particular $\mathrm{KH}$ and anomalous types, and in the sizes of KH rolled-up vortices and other derived quantities of physical importance for the conditions leading to their formation. Using two-point distant observations and spectral analysis of the tailward magnetic field component, we proposed an alternative method to estimate the wavelength and phase speed at a single spacecraft from a statistical fit to the data at the other site. This method might be further validated with more observations.

[23] Acknowledgments. C. Foullon acknowledges financial support from the UK Science and Technology Facilities Council (STFC) on the CFSA Rolling Grant. C. J. Farrugia acknowledges financial support from NASA grant NNX08AD11G. We acknowledge use of Geotail and Cluster data, in particular from magnetic field in situ experiments, as shown in this paper, and thank their respective Principal Investigators, namely S. Kokubun (Geotail/MGF) and E. Lucek (Cluster/FGM).

[24] Masaki Fujimoto thanks Johan De Keyser and another reviewer for their assistance in evaluating this paper.

\section{References}

Bai, T., and E. W. Cliver (1990), A 154 day periodicity in the occurrence rate of proton flares, Astrophys. J., 363, 299-309, doi:10.1086/169342.

Balogh, A., et al. (2001), The Cluster Magnetic Field Investigation: Overview of in-flight performance and initial results, Ann. Geophys., 19, 1207-1217.

Belmont, G., and G. Chanteur (1989), Advances in magnetopause KelvinHelmholtz instability studies, Phys. Scr., 40, 124-128.

Chandrasekhar, S. (1961), Hydrodynamic and Hydromagnetic Stability, Clarendon, Oxford, U. K

Chen, S.-H., and M. G. Kivelson (1993), On nonsinusoidal waves at the Earth's magnetopause, Geophys. Res. Lett., 20, 2699-2702, doi:10.1029/93GL02622.

Chen, S.-H., M. G. Kivelson, J. T. Gosling, R. J. Walker, and A. J. Lazarus (1993), Anomalous aspects of magnetosheath flow and of the shape and oscillations of the magnetopause during an interval of strongly northward interplanetary magnetic field, J. Geophys. Res., 98, 5727-5742.

De Keyser, J., and M. Roth (2003), Structural analysis of periodic surface waves on the magnetospheric boundary, Planet. Space Sci., 51, 757-768, doi:10.1016/S0032-0633(03)00112-0.

De Keyser, J., G. Gustafsson, M. Roth, F. Darrouzet, M. Dunlop, H. Rème, A. Fazakerley, P. Décréau, and N. Cornilleau-Wehrlin (2004), Reconstruction of the magnetopause and low-latitude boundary layer topology using Cluster multi-point measurements, Ann. Geophys., 22, 2381-2389. De Keyser, J., M. W. Dunlop, C. J. Owen, B. U. Ö. Sonnerup, S. E. Haaland, A. Vaivads, G. Paschmann, R. Lundin, and L. Rezeau (2005), Magnetopause and boundary layer, Space Sci. Rev., 118, 231-320, doi:10.1007/ s11214-005-3834-1.

Elphic, R. C., and C. T. Russell (1979), ISEE-1 and 2 magnetometer observations of the magnetopause, in Magnetospheric Boundary Layers, edited by J. Lemaire, Eur. Space Agency Spec. Publ., 148, 43-50.

Fairfield, D. H., A. Otto, T. Mukai, S. Kokubun, R. P. Lepping, J. T. Steinberg, A. J. Lazarus, and T. Yamamoto (2000), Geotail observations of the Kelvin-Helmholtz instability at the equatorial magnetotail 
boundary for parallel northward fields, J. Geophys. Res., 105, 21,159-21,174, doi:10.1029/1999JA000316.

Fairfield, D. H., M. M. Kuznetsova, T. Mukai, T. Nagai, T. I. Gombosi, and A. J. Ridley (2007), Waves on the dusk flank boundary layer during very northward interplanetary magnetic field conditions: Observations and simulation, J. Geophys. Res., 112, A08206, doi:10.1029/2006JA012052.

Farrugia, C. J., F. T. Gratton, L. Bender, H. K. Biernat, N. V. Erkaev, J. M. Quinn, R. B. Torbert, and V. Dennisenko (1998), Charts of joint Kelvin-Helmholtz and Rayleigh-Taylor instabilites at the dayside magnetopause for strongly northward interplanetary magnetic field, J. Geophys. Res., 103, 6703-6728, doi:10.1029/97JA03248.

Farrugia, C. J., et al. (2000), Coordinated Wind, Interball/tail, and ground observations of Kelvin-Helmholtz waves at the near-tail, equatorial magnetopause at dusk: January 11, 1997, J. Geophys. Res., 105, 7639-7668, doi:10.1029/1999JA000267.

Foullon, C., C. J. Owen, S. Dasso, L. M. Green, I. Dandouras, H. A. Elliott, A. N. Fazakerley, Y. V. Bogdanova, and N. U. Crooker (2007), Multispacecraft study of the 21 January 2005 ICME: Evidence of current sheet substructure near the periphery of a strongly expanding, fast magnetic cloud, Sol. Phys., 244, 139-165, doi:10.1007/s11207-007-0355-y.

Foullon, C., C. J. Farrugia, A. N. Fazakerley, C. J. Owen, F. T. Gratton, and R. B. Torbert (2008), Evolution of Kelvin-Helmholtz activity on the dusk flank magnetopause, J. Geophys. Res., 113, A11203, doi:10.1029/ 2008JA013175.

Foullon, C., C. J. Farrugia, A. N. Fazakerley, C. J. Owen, F. T. Gratton, and R. B. Torbert (2009), Reply to comment by H. Hasegawa on "Evolution of Kelvin-Helmholtz activity on the dusk flank magnetopause", J. Geophys. Res., 114, A10201, doi:10.1029/2009JA014444.

Foullon, C., C. J. Farrugia, C. J. Owen, A. N. Fazakerley, and F. T. Gratton (2010), Kelvin-Helmholtz multi-spacecraft studies at the Earth's magnetopause boundaries, in 12th Solar Wind Conference, edited by M. Maksimovic et al., AIP Conf. Proc., 1216, 483-486.

Fujimoto, M., and T. Terasawa (1994), Anomalous ion mixing within an MHD scale Kelvin-Helmholtz vortex, J. Geophys. Res., 99, 8601-8613.

Hasegawa, A. (1975), Plasma Instabilities and Nonlinear Effects, Springer, New York.

Hasegawa, H. (2009), Comment on "Evolution of Kelvin-Helmholtz activity on the dusk flank magnetopause" by Foullon et al., J. Geophys. Res., 114, A03205, doi:10.1029/2008JA013887.

Hasegawa, H., M. Fujimoto, T.-D. Phan, H. Rème, A. Balogh, M. W. Dunlop, C. Hashimoto, and R. TanDokoro (2004), Transport of solar wind into Earth's magnetosphere through rolled-up Kelvin-Helmholtz vortices, Nature, 430, 755-758, doi:10.1038/nature02799.

Hasegawa, H., et al. (2009), Kelvin-Helmholtz waves at the Earth's magnetopause: Multiscale development and associated reconnection, J. Geophys. Res., 114, A12207, doi:10.1029/2009JA014042.

Hones, E. W., Jr., S. J. Bame, J. R. Asbridge, J. Birn, G. Paschmann, N. Sckopke, and G. Haerendel (1981), Further determination of the characteristics of magnetospheric plasma vortices with Isee 1 and 2 , J. Geophys. Res., 86, 814-820, doi:10.1029/JA086iA02p00814.

Kokubun, S., T. Yamamoto, M. H. Acuna, K. Hayashi, K. Shiokawa, and H. Kawano (1994), The Geotail magnetic field investigation, J. Geomagn. Geoelectr., 46, 7-21.

Lavraud, B., J. E. Borovsky, A. J. Ridley, E. W. Pogue, M. F. Thomsen, H. Rème, A. N. Fazakerley, and E. A. Lucek (2007), Strong bulk plasma acceleration in Earth's magnetosheath: A magnetic slingshot effect?, Geophys. Res. Lett., 34, L14102, doi:10.1029/2007GL030024.

Manuel, J. R., and J. C. Samson (1993), The spatial development of the low-latitude boundary layer, J. Geophys. Res., 98, 17,367-17,386, doi:10.1029/93JA01524.

Mills, K. J., A. W. Longbottom, A. N. Wright, and M. S. Ruderman (2000), Kelvin-Helmholtz instability on the magnetospheric flanks: An absolute and convective instability approach, J. Geophys. Res. 105, 27,685-27,700, doi:10.1029/1999JA000289.

Miura, A. (1984), Anomalous transport by magnetohydrodynamic KelvinHelmholtz instabilities in the solar wind-magnetosphere interaction, J. Geophys. Res., 89, 801-818.
Miura, A. (1999a), A quantitative test of the self-organization hypothesis of the magnetopause Kelvin-Helmholtz instability as an inverse problem, Geophys. Res. Lett., 26, 409-412, doi:10.1029/1998GL900300.

Miura, A. (1999b), Self-organization in the two-dimensional magnetohydrodynamic transverse Kelvin-Helmholtz instability, J. Geophys. Res., 104, 395-412, doi:10.1029/98JA02530.

Miura, A., and P. L. Pritchett (1982), Nonlocal stability analysis of the MHD Kelvin-Helmholtz instability in a compressible plasma, J. Geophys. Res., 87, 7431-7444.

Nykyri, K., and A. Otto (2004), Influence of the Hall term on KH instability and reconnection inside KH vortices, Ann. Geophys., 22, 935-949.

Ogilvie, K. W., and R. J. Fitzenreiter (1989), The Kelvin-Helmholtz instability at the magnetopause and inner boundary layer surface, J. Geophys. Res., 94, 15,113-15,123, doi:10.1029/JA094iA11p15113.

Otto, A., and D. H. Fairfield (2000), Kelvin-Helmholtz instability at the magnetotail boundary: MHD simulation and comparison with Geotail observations, J. Geophys. Res., 105, 21,175-21,190, doi:10.1029/1999JA000312.

Owen, C. J., M. G. G. T. Taylor, I. C. Krauklis, A. N. Fazakerley, M. W. Dunlop, and J. M. Bosqued (2004), Cluster observations of surface waves on the dawn flank magnetopause, Ann. Geophys., 22, 971-983.

Phan, T. D., et al. (1997), Low-latitude dusk flank magnetosheath, magnetopause, and boundary layer for low magnetic shear: Wind observations, J. Geophys. Res., 102, 19,883-19,896.

Roelof, E. C., and D. G. Sibeck (1993), Magnetopause shape as a bivariate function of interplanetary magnetic field $B_{z}$ and solar wind dynamic pressure, J. Geophys. Res., 98, 21,421-21,450.

Russell, C. T., M. M. Mellott, E. J. Smith, and J. H. King (1983), Multiple spacecraft observations of interplanetary shocks Four spacecraft determination of shock normals, J. Geophys. Res., 88, 4739-4748.

Saunders, M. A., D. J. Southwood, T. A. Fritz, and E. W. Hones Jr. (1983), Hydromagnetic vortices. I - The 11 December 1977 event, Planet. Space Sci., 31, 1099-1116, doi:10.1016/0032-0633(83)90098-3.

Schwartz, S. J. (1998), Shock and discontinuity normals, Mach numbers, and related parameters, in Analysis Methods for Multi-Spacecraft Data, edited by G. Pashmann and P. W. Daly, chap. 10, pp. 249-270, Int. Space Sci. Inst., Bern, Switzerland.

Southwood, D. J. (1968), The hydromagnetic stability of the magnetospheric boundary, Planet. Space Sci., 16, 587-605, doi:10.1016/00320633(68)90100-1.

Thomas, V. A., and D. Winske (1993), Kinetic simulations of the Kelvin-Helmholtz instability at the magnetopause, J. Geophys. Res., 98 , $11,425-11,438$.

Torrence, C., and G. P. Compo (1998), A practical guide to wavelet analysis, Bull. Am. Meteorol. Soc., 79(1), 61-78.

Walker, A. D. M. (1981), The Kelvin-Helmholtz instability in the lowlatitude boundary layer, Planet. Space Sci., 29, 1119-1133, doi:10.1016/ 0032-0633(81)90011-8.

Wright, A. N., K. J. Mills, M. S. Ruderman, and L. Brevdo (2000), The absolute and convective instability of the magnetospheric flanks, J. Geophys. Res., 105, 385-394, doi:10.1029/1999JA900417.

Wu, C. C. (1986), Kelvin-Helmholtz instability at the magnetopause boundary, J. Geophys. Res., 91, 3042-3060.

C. J. Farrugia and R. B. Torbert, Space Science Center, Department of Physics, University of New Hampshire, Durham, NH 03824, USA.

A. N. Fazakerley and C. J. Owen, Mullard Space Science Laboratory, University College London, Holmbury St Mary, Dorking, Surrey RH5 6NT, UK.

C. Foullon, Center for Fusion, Space and Astrophysics, Department of Physics, University of Warwick, Coventry CV4 7AL, UK.

F. T. Gratton, Instituto de Física del Plasma, CONICET, Universidad de Buenos Aires, Pab. 1, 1428, Buenos Aires, Argentina. 